\title{
Distinct requirements of Autophagy-related genes in programmed cell death
}

\author{
T Xu ${ }^{1}$, S Nicolson ${ }^{1}$, D Denton ${ }^{* 1}$ and S Kumar* ${ }^{* 1}$
}

Although most programmed cell death (PCD) during animal development occurs by caspase-dependent apoptosis, autophagydependent cell death is also important in specific contexts. In previous studies, we established that PCD of the obsolete Drosophila larval midgut tissue is dependent on autophagy and can occur in the absence of the main components of the apoptotic pathway. As autophagy is primarily a survival mechanism in response to stress such as starvation, it is currently unclear if the regulation and mechanism of autophagy as a pro-death pathway is distinct to that as pro-survival. To establish the requirement of the components of the autophagy pathway during cell death, we examined the effect of systematically knocking down components of the autophagy machinery on autophagy induction and timing of midgut PCD. We found that there is a distinct requirement of the individual components of the autophagy pathway in a pro-death context. Furthermore, we show that TORC1 is upstream of autophagy induction in the midgut indicating that while the machinery may be distinct the activation may occur similarly in PCD and during starvation-induced autophagy signalling. Our data reveal that while autophagy initiation occurs similarly in different cellular contexts, there is a tissue/function-specific requirement for the components of the autophagic machinery.

Cell Death and Differentiation (2015) 22, 1792-1802; doi:10.1038/cdd.2015.28; published online 17 April 2015

There is a fundamental requirement for multicellular organisms to remove excess, detrimental, obsolete and damaged cells by programmed cell death (PCD). ${ }^{1,2}$ In the majority of cases caspase-dependent apoptosis is the principle pathway of PCD; however, there are other modes of cell death with important context-specific roles, such as autophagy. ${ }^{3,4}$ Defects in autophagy have significant adverse consequences to normal cellular functions and contribute to the pathogenesis of numerous human diseases. This is particularly evident in cancer where depending on the context autophagy can have tumour-suppressing or -promoting roles. Given the number of clinical trials targeting autophagy in cancer therapy, it will be critically important to understand the context-specific regulation and functions of autophagy. ${ }^{5}$

Autophagy is a highly conserved multi-step catabolic process characterised by the encapsulation of part of the cytoplasm inside a double-membrane vesicle called the autophagosome. Autophagosomes then fuse with lysosomes and the components are subsequently degraded by acidic lysosomal hydrolases. ${ }^{6}$ The process of autophagy can be functionally divided into four groups: (1) serine/threonine kinase Atg1 (ULK1 in mammals) complex and its regulators responsible for the induction of autophagy; (2) the class III phosphatidylinositol 3-kinase (PI3K) complex, which involves Atg6 and functions in the nucleation of the autophagosome; (3) the Atg8 and Atg12 conjugation systems, which involves several Autophagy-related (Atg) proteins essential for the expansion of autophagosome; and (4) Atg9 and its associated proteins including Atg2 and Atg18, which aids the recycling of lipid and proteins. ${ }^{7}$ In addition, several of the Atg proteins can function in multiple steps. For example, Atg1 interacts with proteins with different functions (e.g. Atg8, Atg18 and others), suggesting that it is not only required for initiation but also participates in the formation of autophagosomes. ${ }^{8}$ It is yet to be fully established if the context-specific functions of autophagy have distinct requirements for select components of the autophagy pathway.

High levels of autophagy are induced in response to stress, such as nutrient deprivation, intracellular stress, high temperature, high culture density, hormones and growth factor deprivation. ${ }^{9,10}$ The target of rapamycin (TOR) pathway is a central mediator in regulating the response to nutrients and growth signalling. TOR functions in two distinct complexes, with regulatory associated protein of TOR (Raptor) in TOR complex 1 (TORC1) or with rapamycin insensitive companion of TOR (Rictor) in TOR complex 2 (TORC2). ${ }^{11-15}$ Of these, TORC1 regulates autophagy; in nutrient-rich conditions, TORC1 activity inhibits the Atg1 complex preventing autophagy and cellular stress such as starvation leads to inactivation of TORC1 promoting a dramatic increase in autophagy. TORC2 can also negatively regulate autophagy via the $\mathrm{FoxO} 3$ complex in specific context. ${ }^{16}$

Most direct in vivo evidence for a role of autophagy in cell death has emerged from studies in Drosophila. ${ }^{5}$ Developmentally regulated removal of the Drosophila larval midgut can occur in the absence of canonical apoptosis pathway, whereas inhibiting autophagy delays the process. ${ }^{17,18}$ Also, inhibition of autophagy leads to delayed degradation of larval salivary glands in Drosophila. ${ }^{19}$ Genetic studies have shown that many of the Atg genes known to be involved in starvation-induced

${ }^{1}$ Centre for Cancer Biology, University of South Australia, Adelaide, SA 5001, Australia

${ }^{*}$ Corresponding author: D Denton or S Kumar, Centre for Cancer Biology, University of South Australia, Frome Road, Adelaide, SA 5001, Australia. Tel: +61 88222 3738; Fax: +61 88222 3162; E-mail: donna.denton@unisa.edu.au or sharad.kumar@unisa.edu.au

Abbreviations: Atg, autophagy-related; PCD, programmed cell death; PI3K, phosphatidylinositol 3-kinase; RPF, relative to puparium formation; TOR, target of rapamycin Received 31.10.14; revised 06.2.15; accepted 23.2.15; Edited by M Piacentini; published online 17.4.15 
autophagy in the Drosophila fat body are also involved in autophagy-dependent degradation of salivary glands and midgut. 5,20,21 However, systematic studies to test whether starvation-induced autophagy and autophagy required for PCD require identical components have not been carried out, and there are some observations suggesting that there may be distinctions. For example, in Atg7-null mutants autophagy is perturbed but the larval-adult midgut transition proceeds normally. $^{22}$ In addition, a novel Atg7- and Atg3-independent autophagy pathway is required for cell size reduction during midgut removal. ${ }^{23}$ Here we show that downregulation of TORC1 activity is required for induction of autophagy during midgut removal. Surprisingly, however, the requirement of part of the autophagy machinery during midgut degradation was found to be distinct to that which is required during autophagy induced by starvation. We report that $A$ tg genes required for autophagy initiation, Atg8a and recycling are all essential for autophagy-dependent midgut removal, whereas other components of the elongation and nucleation steps are not essential.

\section{Results}

We have previously shown that knockdown of $\operatorname{Atg} 1$ and $\operatorname{Atg} 18$ or Atg2 and Atg18 mutants blocks autophagy and significantly delays larval midgut removal. ${ }^{17}$ However, it is unknown whether the signals that regulate this are similar to those regulating induction of autophagy in response to starvation and whether the canonical autophagy machinery components are required for this process. Here we dissect out the requirement of the components of the autophagy pathway by systematically knocking down Atg genes in the midgut and examining the effect on autophagy induction and midgut removal. The level of knockdown for each of the RNAi lines used was assessed by real-time qPCR and in all cases the lines that gave the best knockdown for the gene were selected for analyses (Supplementary Figure S1).

Autophagy induction: the Atg1 complex and its regulators. A key early step in the initiation of autophagy requires the activity of the Atg1 complex, comprising Atg1, Atg13, Atg17 (FIP200 in mammals) and Atg101. ${ }^{24-26}$ Similarly in Drosophila, Atg1 activity also requires Atg13, Atg17 and Atg101 in the complex. ${ }^{27,28}$ Our previous work has shown that Atg1 is essential for autophagy and midgut removal. ${ }^{17}$ To determine the requirements of other subunits of the Atg1 complex in autophagy during midgut cell death, we examined the effects of knockdown of Atg13, Atg17 and Atg101. Similar to Atg1, depletion of Atg13, Atg17 or Atg101 resulted in significantly delayed midgut degradation (Figure 1). At the onset of PCD ( $0 \mathrm{~h}$ relative to puparium formation (RPF)) and during PCD when the gastric caeca have contracted in the control animals (+4 h RPF), Atg1, Atg13, Atg17 or Atg101 knockdown resulted in larger midguts (Figures 1a-f). The levels of pmCherry-Atg8a puncta were dramatically reduced upon knockdown of $\operatorname{Atg} 1, \operatorname{Atg} 13, \operatorname{Atg} 17$ or $\operatorname{Atg} 101$ at $+2 \mathrm{~h}$ RPF compared with the control (Figures 1a-e and g). At later
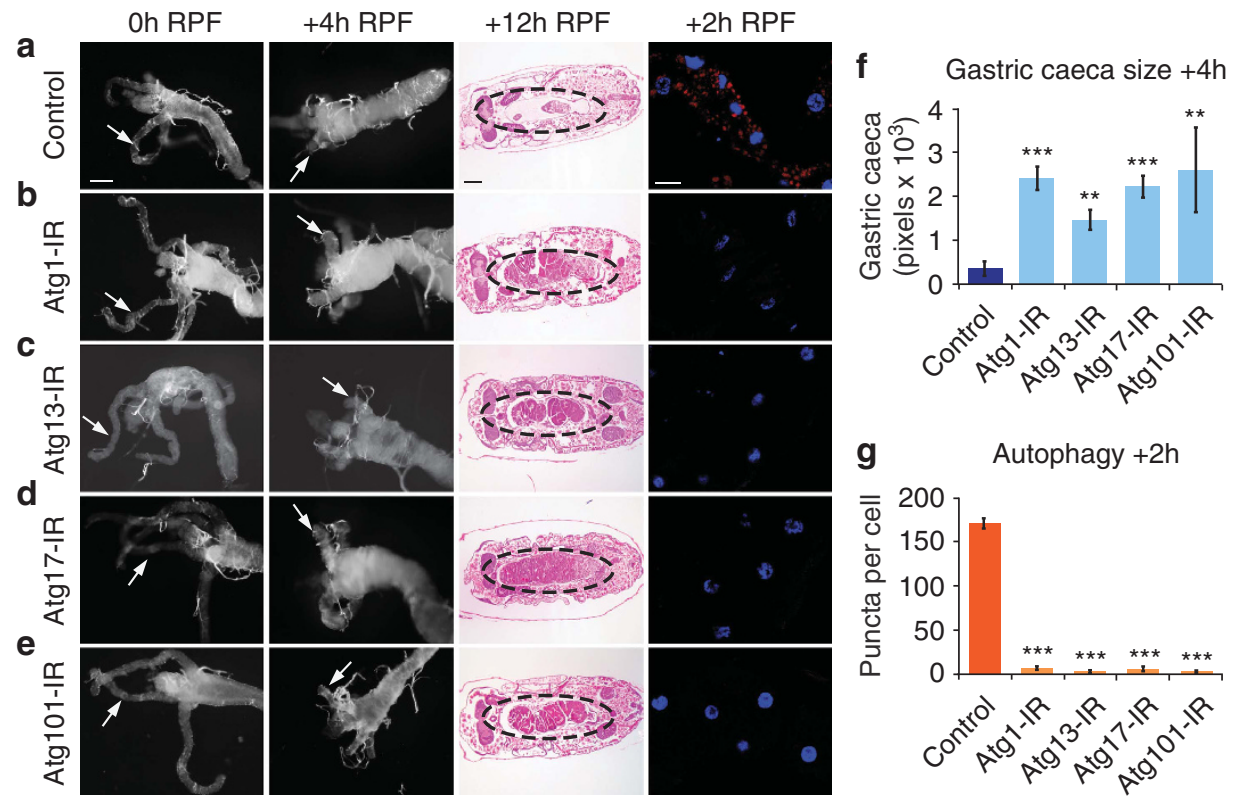

Figure 1 Initiation complex genes are required for autophagy and PCD of the larval midgut. (a-e) Morphology of midguts (left) at $0 \mathrm{~h}$ RPF and $+4 \mathrm{~h}$ RPF showing the contraction of gastric caeca (arrow) as an indicator of midgut degradation. Compared with (a) control (NP1-GAL4/+; pmCherry-Atg8a/+) the knockdown of (b) Atg1-IR (NP1GAL4/+; UAS-Atg1 RNAi/pmCherry-Atg8a), (c) Atg13-IR(NP1-GAL4/+; UAS-Atg13 RNAi/pmCherry-Atg8a), (d) Atg17-IR (NP1-GAL4/ UAS-Atg17 RNAi; +/pmCherry-Atg8a) and (e) Atg101-IR (NP1-GAL4/+; UAS-Atg101 RNAi/pmCherry-Atg8a) shows large midgut with persisting gastric caeca at both $0 \mathrm{~h} \mathrm{RPF}$ and $+4 \mathrm{~h}$ RPF. Scale bar represents $200 \mu \mathrm{m}$. Histological analysis of paraffin sections at $+12 \mathrm{~h} \mathrm{RPF}$ shows large persisting midgut (circled) in (b) Atg1-IR, (c) Atg13-IR, (d) Atg17-IRand (e) Atg101-IR, compared to (a) control. Scale bar represents $200 \mu \mathrm{m}$. (f) Quantitation of gastric caeca size (average pixels \pm S.D.) at $+4 \mathrm{~h} \mathrm{RPF}$ from (a-e) $\left(N \geq 10,{ }^{* *} P<0.01,{ }^{* * *} P<0.001\right)$. (a-e, right) Autophagy puncta examined using pmCherry-Atg8a (red) and DNA stained with Hoechst (blue) in $+2 \mathrm{~h}$ RPF midgut gastric caeca. In (a) control, high pmCherry-Atg8a puncta are observed with (b) Atg1-IR, (c) Atg13-IR, (d) Atg17-IR and (e) Atg101-IR showing undetectable levels. Scale bar represents $10 \mu \mathrm{m}$. (g) The quantitation of the pmCherry-Atg8a puncta as the mean fluorescent pixel intensity per cell at $+2 \mathrm{~h}$ RPF from (a-e, right) using ImageJ, error bars represent S.E.M. $\left(N \geq 10,{ }^{* \star *} P<0.001\right)$ 
developmental stages, the dying midgut continues to condense and at $+12 \mathrm{~h}$ RPF consists of a compact body. Compared with the control at +12 h RPF, pupae from Atg1, Atg13, Atg17 or Atg101 knockdown lines showed dramatically enlarged midgut consistent with delayed removal (Figures 1a-e). The delayed midgut degradation displayed in Atg17 knockdown animals is consistent with recent studies using Atg17 mutants. ${ }^{28}$ Thus our data indicate that all known components of the Atg1 complex are required for Drosophila midgut $\mathrm{PCD}$.

\section{Autophagosome nucleation: class III PI3K complex.} During autophagosome initiation, the class III PI3K complex, consisting of Vps35, Vps15 and Atg6 (Beclin 1 in mammals), generates phosphatidylinositide-3-phosphate required for vesicle nucleation. ${ }^{29}$ Studies in yeast and mammalian cells have revealed that Atg14 controls phosphorylation of Atg6 and mediates the complex localisation to the preautophagosomal structure (PAS). ${ }^{30,31}$ In Drosophila, Atg6, Vps34 (also known as Pi3K59F) and Vps15 (also known as ird1) form a complex required for starvation-induced autophagy in the fat body. ${ }^{32,33}$ However, the function of Atg14 has not been characterised. To assess the role of the class III PI3K complex in midgut PCD, we examined the knockdown of Atg6, Atg14, Vps15 and Vps34. The knockdown of these genes showed midgut removal was affected to differing extents. Surprisingly, knockdown of Atg6 showed normal autophagy levels although the gastric caeca were larger compared with control (Figures 2a and b). This phenotype was also observed in an independent Atg6 knockdown line (Supplementary Figure S2). This is in contrast to Vps15 or Vsp34 knockdown, where the level of autophagy was reduced corresponding to enlargement of gastric caeca (Figures 2d-g). Quantitation of pmCherryAtg8a puncta showed the reduction of autophagy level in Vps15 and Vps34 knockdown was significant (Figure 2g). The knockdown of Atg14 showed only a modest increase in size with a correspondingly modest decrease in autophagy (Figures $2 \mathrm{c}, \mathrm{f}$ and g). A similar result was seen with an Atg14-null mutant (not shown).

Autophagosome expansion: the Atg8 and Atg12 conjugation systems. Expansion of the phagophores into autophgagosomes requires two ubiquitin-like systems: the Atg8-lipid phosphatidylethanolamine (PE) conjugation system and the Atg12-Atg5 system (Figure 3a). Prior to lipidation, cleavage of Atg8 by the cysteine protease, Atg4, allows the E1-like enzyme Atg7 to interact with Atg8. ${ }^{34}$ Following this, Atg8 is then transferred to the E2-like enzyme Atg3 and finally to PE. ${ }^{35}$ Transfer of Atg8 from Atg3 to PE is in part catalysed by the final product of the second conjugation

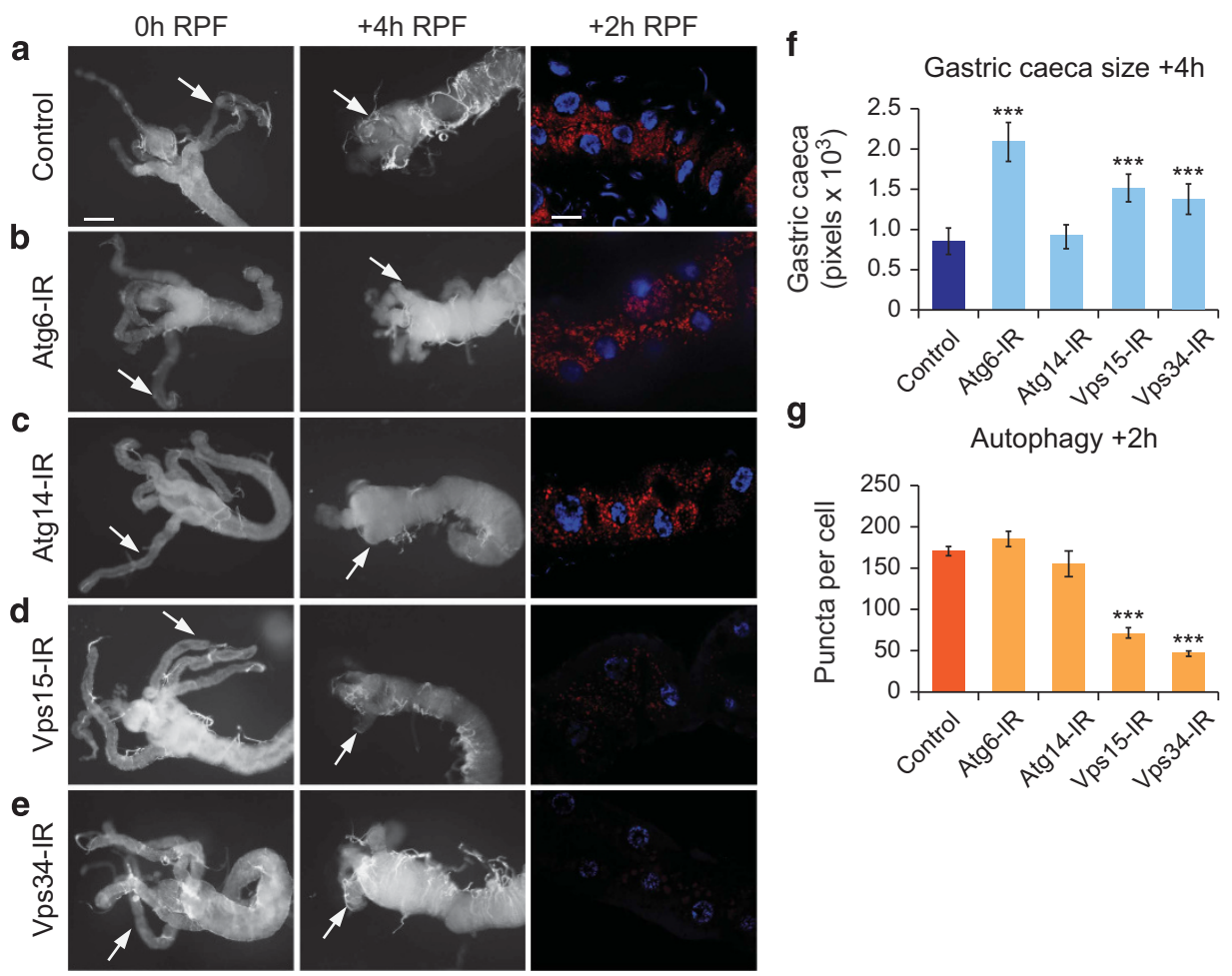

Figure 2 Requirement of the nucleation pathway genes for autophagy and midgut degradation. Compared with the (a) control (NP1-GAL4/+; pmCherry-Atg8a/+), (b) Atg6-IR (NP1-GAL4/+; UAS-Atg6 RNAi/pmCherry-Atg8a), (d) Vps15-IR (NP1-GAL4/+; UAS-Vps15 RNAi/pmCherry-Atg8a), and (e) Vps34-IR (NP1-GAL4/+; UAS-Vps34 RNAi/ pmCherry-Atg8a) have delayed midgut removal shown by reduced contraction of gastric caeca (arrow) whereas (c) Atg14-IR (NP1-GAL4/+; UAS-Atg14 RNAi/pmCherry-Atg8a) is similar to control. Scale bar represents $200 \mu \mathrm{m}$. (f) Quantitation of gastric caeca size (average pixels \pm S.D.) at $+4 \mathrm{~h} R P F$ from $(\mathbf{a}-\mathbf{e})\left(N \geq 10,{ }^{* \star *} P<0.001\right)$. (a-e, right) Autophagy puncta examined using pmCherry-Atg8a (red) and DNA stained with Hoechst (blue) in $+2 \mathrm{~h}$ RPF midgut gastric caeca. In (a) control, (b) Atg6-IR and (c) Atg14-IR similar levels of pmCherry-Atg8a puncta are observed with (d) Vps15-IR and (e) Vps34-IR showing reduced levels. Scale bar represents $10 \mu \mathrm{m}$. (g) The quantitation of the pmCherry-Atg8a puncta as the mean fluorescent pixel intensity per cell at $+2 \mathrm{~h}$ RPF from (a-e) using ImageJ, error bars represent S.E.M. ${ }^{* \star *} P<0.001$ 

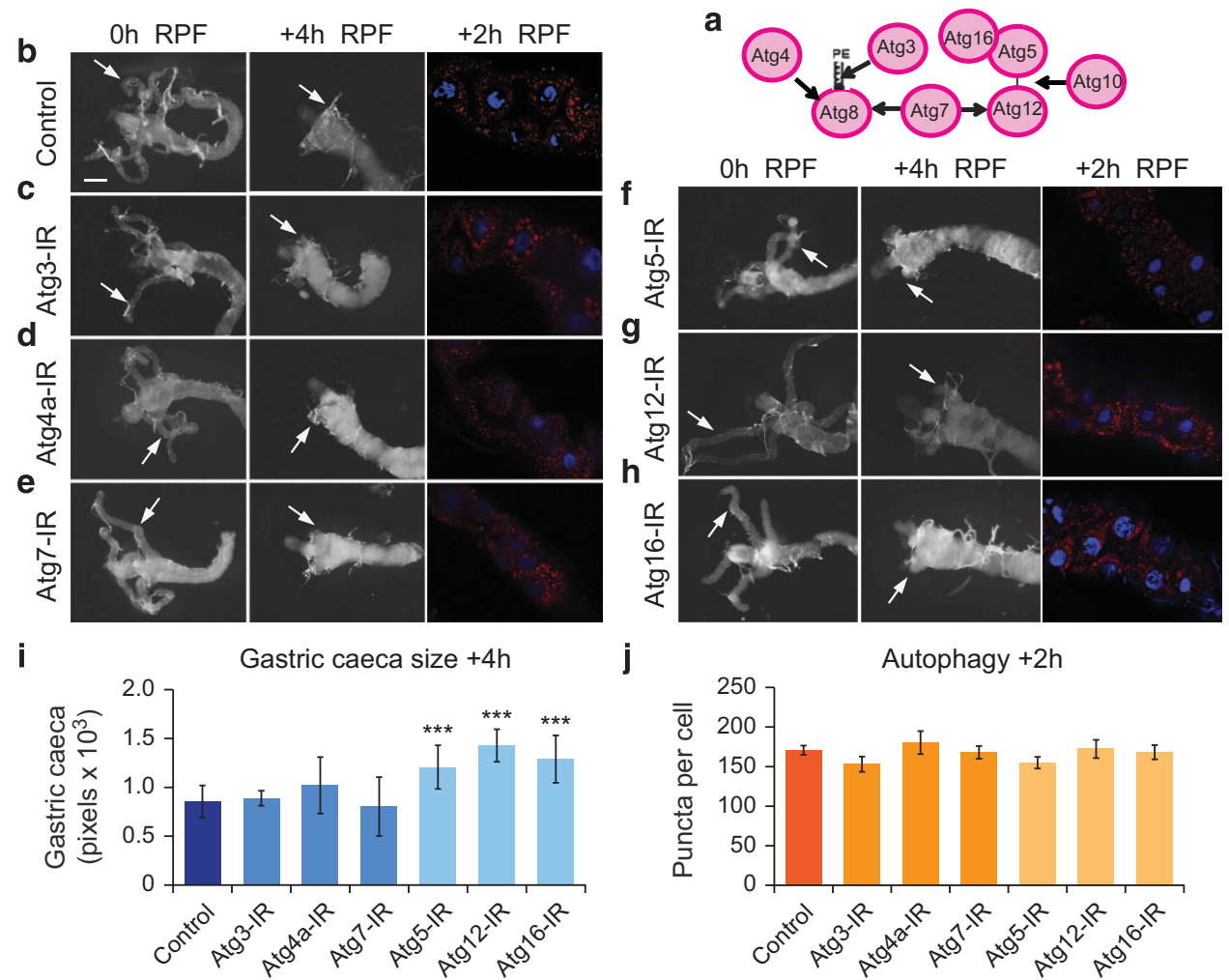

Figure 3 Distinct roles of the conjugation pathways in midgut autophagy and histolysis. (a) Schematic diagram showing the multiple components of the conjugation pathways. Compared with (b) control (NP1-GAL4/+; pmCherry-Atg8a/+), knockdown of (c) Atg3-IR (NP1-GAL4/+; UAS-Atg3 RNAi/pmCherry-Atg8a), (d) Atg4a-IR (NP1-GAL4/+; UAS-Atg4a RNAi/pmCherry-Atg8a) and (e) Atg7-IR (NP1-GAL4/+; UAS-Atg7 RNAi/pmCherry-Atg8a) did not affect gastric caeca contraction at both $0 \mathrm{~h} \mathrm{RPF} \mathrm{(left)} \mathrm{and}+4 \mathrm{~h}$ RPF (middle) (arrow). The knockdown of (f) Atg5-IR (NP1-GAL4/+; UAS-Atg5 RNAi/pmCherry-Atg8a), (g) Atg12-IR (NP1-GAL4/+; UAS-Atg12 RNAi/pmCherry-Atg8a) and (h) Atg16IR (NP1-GAL4/+; UAS-Atg16 RNAi/pmCherry-Atg8a) led to an increase in the size of gastric caeca at both $0 \mathrm{~h} \mathrm{RPF} \mathrm{(left)} \mathrm{and}+4 \mathrm{~h}$ RPF (middle) compared with (b) control. Scale bar represents $200 \mu \mathrm{m}$. (b-h, right) Autophagy puncta examined using pmCherry-Atg8a (red) and DNA stained with Hoechst (blue) in $+2 \mathrm{~h}$ RPF midgut gastric caeca. The level of autophagy at $+2 \mathrm{~h}$ RPF was not affected by knockdown of (c) Atg3-IR, (d) Atg4a-IR, (e) Atg7-IR, (f) Atg5-IR, (g) Atg12-IR or (h) Atg16-IR (right). Scale bar represents 10 $\mu$ m. (i) Quantitation of gastric caeca size (average pixels \pm S.D.) at $+4 \mathrm{~h}$ RPF from $(\mathbf{b}-\mathbf{h})\left(N \geq 10,{ }^{* * \star} P<0.001\right)$. (j) Quantitation of the pmCherry-Atg8a puncta as the mean fluorescent pixel intensity per cell at $+2 \mathrm{~h}$ RPF from $(\mathbf{b}-\mathbf{h})$ using ImageJ, error bars represent S.E.M., $(N \geq 10)$

system, the Atg12-Atg5-Atg16 complex. ${ }^{36}$ Functioning in parallel to the Atg8-PE system, the Atg12-Atg5 conjugation system is similar to that of Atg8-PE system (Figure 3a). The E1- and E2-like enzymes are Atg7 and Atg10, respectively, and mediate the transfer of Atg12 to Atg5. Atg12 is finally conjugated to Atg5, forming an Atg12-Atg5 complex, which associates with Atg16 and assists the elongation of autophagosome. ${ }^{34,36}$

To examine the contribution of the components of the Atg8a-PE conjugation system to autophagy in midgut PCD, we examined the knockdown of Atg3, Atg4a and Atg7. At the onset of PCD ( $0 \mathrm{~h} \mathrm{RPF}$ ) and at a later stage of midgut histolysis (+4 h RPF), depletion of Atg3, Atg4a or Atg7 had no significant effect on midgut removal (Figures $3 \mathrm{~b}-\mathrm{e}$ and i). Consistent with this, high levels of autophagy similar to the control at $+2 \mathrm{~h}$ RPF were observed following Atg3, Atg4a or Atg7 knockdown (Figures $3 \mathrm{~b}-\mathrm{e}$ and j). This suggests that the Atg8-PE conjugation system, Atg3, Atg4a and Atg7, is not essential for autophagy in the midgut. This is in agreement with previous studies that indicate that Atg7 is not essential for midgut removal and that Atg3 and Atg4 are not required for cell size reduction in the midgut. ${ }^{23}$ Together this indicates that lipidation is not the rate-determining step or may not be required for the function of Atg8a in this context.
Interestingly, our analyses of the depletion of components of the Atg12-Atg5-Atg16 complex showed that knockdown of Atg5, Atg12 or Atg16 resulted in a moderate but significant delay in midgut removal (Figures $3 \mathrm{f}-\mathrm{i})$. At the onset (O $\mathrm{h}$ RPF) and during (+4 h RPF) PCD, Atg5, Atg12 or Atg16 knockdown midguts were larger than the control (Figures $3 \mathrm{~b}$ and $\mathrm{f}-\mathrm{i}$ ). This is consistent with the findings that clones of cells depleted for Atg5, Atg12 or Atg16 are larger than the neighbouring control cells. ${ }^{23}$ Surprisingly, however, examination of autophagy levels in the Atg5, Atg12 or Atg16 knockdown showed no significant difference in pmCherry-Atg8a puncta at $+2 \mathrm{~h}$ RPF (Figures 3f-h and j). An additional marker of autophagy commonly used in Drosophila is lysotracker staining, and we observed overlap between lysotracker staining and pmCherry-Atg8a puncta in dying midguts (Supplementary Figure S3). Lysotracker staining of Atg5, Atg12 or Atg16 knockdown midguts as an alternative marker of autophagy did not show any obvious differences compared with the control, whereas they were dramatically different to Atg1 knockdown that blocks autophagy (Supplementary Figure S3).

Given that the knockdown of components of the Atg8a conjugation system had no dramatic effect on autophagy and midgut removal, we examined the contribution of Atg8a to midgut autophagy and PCD. There are two Atg8 paralogues 
annotated in the Drosophila genome, Atg8a and Atg8b. During the late larval stage, developmental autophagy is induced in the fat body with increased expression of both Atg8a and Atg $8 b .^{37}$ Similarly, Atg8a and Atg8b are transcriptionally induced during starvation in the fat body. ${ }^{8,38}$ During midgut histolysis, the expression level of Atg8a is high compared with very low expression of Atg8b (data not shown). Consistent with the central role of Atg8a in autophagy, both Atg8a knockdown and mutant show dramatically delayed midgut removal and loss of autophagy (Figures $4 a-c$, e and f). At the onset of midgut removal ( $0 \mathrm{~h} \mathrm{RPF)}$ and during histolysis ( $+4 \mathrm{~h}$ RPF), the Atg8a-depleted midguts were significantly larger than the controls (Figure $4 a-c$ and e). The level of autophagy puncta was detected using an alternative marker, GFP-Atg5, which showed a block in autophagy in the Atg8a knockdown (Figures $4 a, b$ and f). Similarly, autophagy puncta was significantly reduced in the Atg8a mutant midguts (Figures $4 \mathrm{c}$ and $\mathrm{f}$ ). These data indicate that Atg8a has an essential role in midgut removal.

Selection of autophagic cargo can be determined by cargo receptors that interact with Atg8 on the isolation membrane. The multifunctional scaffold protein p62 (also known as SQSTM1 in mammals) binds ubiquitinated proteins and acts as a cargo receptor by binding Atg8 targeting ubiquitinated proteins for degradation by autophagy. ${ }^{39}$ The Drosophila p62 orthologue ref(2)P associates with ubiquitin-positive protein aggregates and accumulates when autophagy is blocked. ${ }^{40}$ Knockdown of ref(2)P in the midgut did not affect autophagy or midgut removal (Figures 4d-f), suggesting that it is not essential for autophagy-dependent PCD.

Preautophagosomal cycling complex: Atg9, Atg2 and Atg18. The transmembrane protein Atg9 is part of the core autophagy machinery and is required for autophagosome formation but is absent from the completed vesicle. ${ }^{29,41,42}$ There are two sources of Atg9, the intercellular pools of Atg9 and the Atg9 on the PAS. ${ }^{43}$ The recycling of Atg9 from the PAS to the intracellular pools is thought to be regulated by three protein complexes, including Atg1-Atg13, Atg2-Atg18 and the PI3K complex. All three complexes are required for effective bulk autophagy. ${ }^{25,44,45}$ The Atg1-Atg13 and the class III PI3K complexes, as described above, have a role in the early steps of autophagosome biogenesis as well, where they regulate the initial step of the double-membrane vesicle formation. ${ }^{46}$ Two functions for the Atg9 complex have been proposed; by reversibly binding to another Atg9 molecule it can both promote lipid transport to the PAS to facilitate nucleation, and also act to promote the assembly of an intact autophagosome membrane. ${ }^{43}$

Consistent with and in addition to our previous studies, the knockdown of Atg2, Atg9 and Atg18 resulted in delayed midgut removal (Figures $5 \mathrm{a}-\mathrm{e})$. The midguts at the onset ( $\mathrm{h}$ RPF) and during histolysis ( $+4 \mathrm{~h}$ RPF) were significantly larger than the control and at a later time point (+12 h RPF) the midgut in the knockdown animals persisted while it was condensed in control (Figures $5 \mathrm{a}-\mathrm{e}$ ). Examination of autophagy puncta at $+2 \mathrm{~h}$ RPF

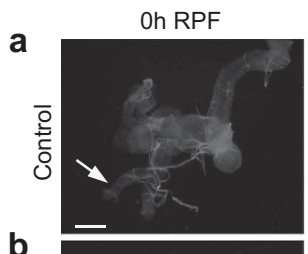

b

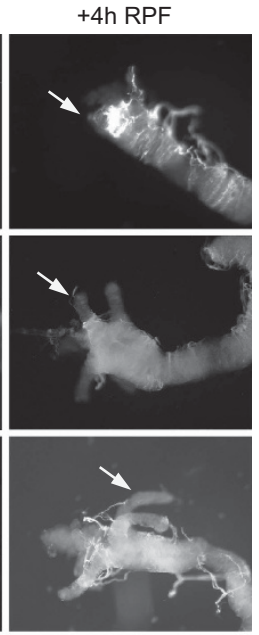

d

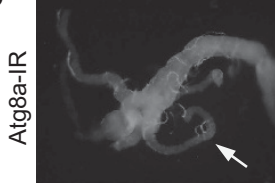

c
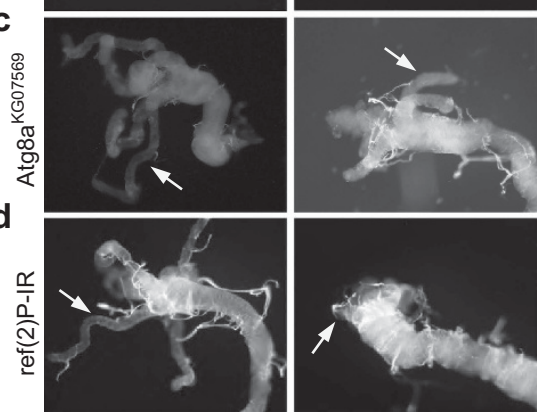
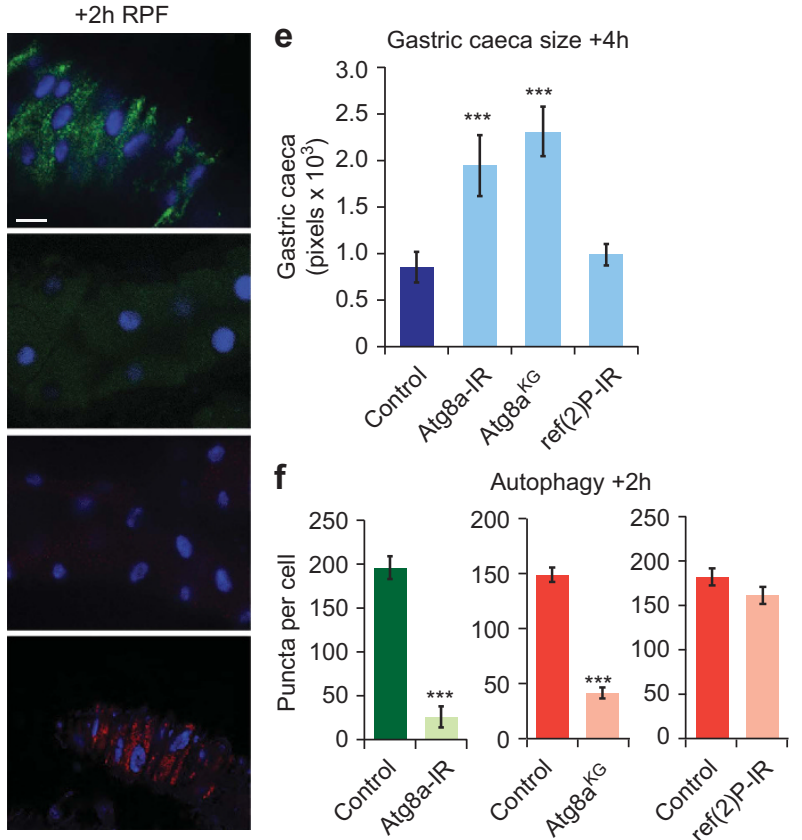

Figure 4 Atg8a is required for autophagy and PCD of the midgut while ref(2)P is not. Morphology of midguts at $0 \mathrm{~h} \mathrm{RPF} \mathrm{(left)} \mathrm{and}+4 \mathrm{~h} \mathrm{RPF} \mathrm{(middle)} \mathrm{comparing} \mathrm{(a)} \mathrm{control}$ (NP1-GAL4/+; UAS-eGFP-Atg5/+) with (b) Atg8a-IR (NP1-GAL4/+; UAS-Atg8a RNAi/UAS-eGFP-Atg5) and (c) Atg8a $a^{K G 07569}$ (Atg8a ${ }^{K G 07569} /$ Y; +; pmCherryAtg8a/+) shows increased size of midgut with larger gastric caeca at both $0 \mathrm{~h}$ RPF and $+4 \mathrm{~h}$ RPF (left and middle, arrows). (d) ref(2)P-IR (NP1-GAL4/+; UAS-ref(2P) RNAi/ pmCherry-Atg8a) was similar to the control. Scale bar represents $200 \mu \mathrm{m}$. (a and $\mathbf{b}$, right) Autophagy induction monitored by eGFP-Atg5 puncta at $+2 \mathrm{~h}$ RPF and DNA stained by Hoechst (blue). Scale bar represents $10 \mu \mathrm{m}$. The level of autophagy in (b) Atg8a-IR is dramatically lower than the control (a, right). (c, right) Similarly, Atg8a mutant showed decreased autophagy. (d, right) ref(2)P-IR showed no effect on autophagy. (e) Quantitation of gastric caeca size (average pixels \pm S.D.) at $+4 \mathrm{~h}$ RPF from (a-d, middle) $\left(N \geq 10,{ }^{* * *} P<0.001\right)$. (f) Quantitation of puncta per cell (eGFP-Atg5 for Atg8a-IR; pmCherry-Atg8a for Atg8 ${ }^{K G 07569}$ and ref(2)P-IR) compared with control at $+2 \mathrm{~h}$ RPF. Error bars represent S.E.M. $\left(N \geq 10,{ }^{* \star *} P<0.001\right)$ 


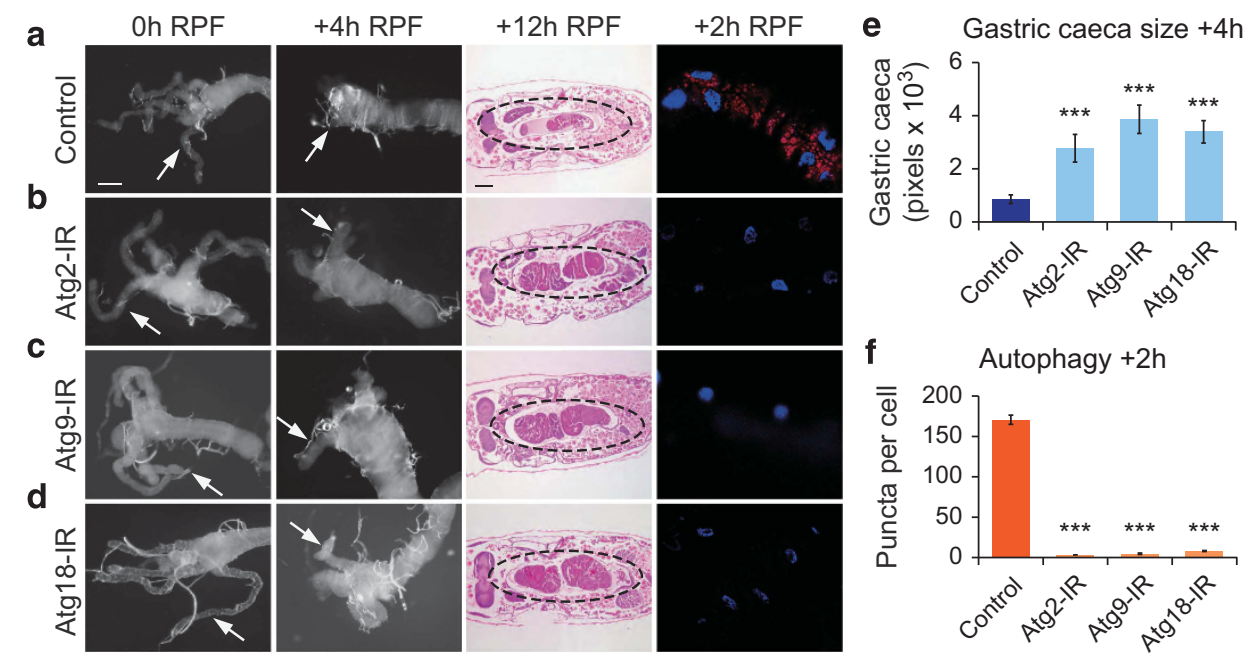

Figure 5 Knockdown of Atg2, Atg9 and Atg18 dramatically reduces autophagy and severely delays midgut PCD. Morphology of midguts at $0 \mathrm{~h} \mathrm{RPF}$ and $+4 \mathrm{~h}$ RPF comparing (a) control (NP1-GAL4/+; pmCherry-Atg8a/+) with (b) Atg2-IR (NP1-GAL4/+; UAS-Atg2 RNAi/pmCherry-Atg8a), (c) Atg9-IR (NP1-GAL4/+; UAS-Atg9 RNAi/pmCherry-Atg8a) and (d) Atg18-IR (NP1-GAL4/+; UAS-Atg18 RNAi/pmCherry-Atg8a), shows a strong delay in midgut contraction (arrow). Scale bar represents $200 \mu \mathrm{m}$. Histology of pupal sections at $+12 \mathrm{~h}$ RPF shows persisting midgut (circled) in (b) Atg2-IR, (c) Atg9-IR and (d) Atg18-IR. Scale bar represents $200 \mu$ m. Autophagy marker pmCherry-Atg8a (red) is significantly reduced in (b) Atg2-IR, (c) Atg9-IR and (d) Atg18-IR compared with the control (a). DNA is stained by Hoechst (blue). Scale bar represents $10 \mu \mathrm{m}$. (e) Quantitation of gastric caeca size (average pixels \pm S.D.) at $+4 \mathrm{~h} R P F$ from $(\mathbf{a}-\mathbf{d})\left(N \geq 10,{ }^{* * *} P<0.001\right)$. (f) Quantitation of pmCherry-Atg8a puncta at $+2 \mathrm{~h}$ RPF from (a-d) (average puncta/cell error bars represent S.E.M., $\left.{ }^{\star \star *} P<0.001\right)$

showed a significant block in autophagy induction in the Atg2, Atg9 and Atg18 knockdown (Figures 5a-d and f). These results suggest an essential role of Atg2, Atg9 and Atg18 in autophagy and midgut degradation.

Autophagy induction in response to starvation in the fat body. The Drosophila fat body undergoes rapid autophagy induction following starvation, and several Atg genes, including, Atg1, Atg3, Atg5, Atg7, Atg12 and Atg18, have been shown to function during starvation-induced autophagy in the fat body. ${ }^{8,41}$ Given our findings that not all Atg genes are required for autophagy during midgut removal, we examined the effect of their knockdown in the fat body to determine whether the Atg genes are required during starvation-induced autophagy in this tissue. Knockdown of Atg genes in the fat body reveals that genes essential for autophagy during midgut removal are also required for starvation-induced autophagy in the fat body (Figure 6). Whereas Atg8a conjugation pathway genes (Atg3 and Atg7), Atg12 conjugation genes (Atg5, Atg12 and Atg16) and Atg6, which are not required for autophagy during midgut removal, are required for starvation-induced autophagy in the fat body (Figure 6). Taken together our data, directly comparing the core components of autophagy in midgut PCD and starvation-induced autophagy, provide strong in vivo evidence that the requirements of the autophagic machinery are distinct in specific tissues and contexts.

Downregulation of TORC1 is required for midgut removal. TORC1 is a crucial negative regulator of autophagy, and down regulation of TORC1 signalling promotes the activation of the autophagy initiation complex. ${ }^{47}$ Knockdown of Tor using two independent lines induced premature autophagy in the midgut and resulted in shortened gastric caeca at an early stage ( $-4 \mathrm{~h}$ RPF) (Figures $7 \mathrm{a}, \mathrm{b}$, e and $\mathrm{f}$ and Supplementary Figure S4). The severity of the phenotype (both the level of autophagy and gastric caeca size) correlated with the level of knockdown (Supplementary Figure S4). As rapamycin is a commonly used inhibitor of Tor, we fed larvae with food that contained rapamycin. Lysotracker staining shows that, following $2 \mathrm{~h}$ of rapamycin feeding to $96 \mathrm{~h}$ after egg deposition (AED) larvae, autophagy was induced in both fat body and midgut (Figure 7k). This further implies that Tor is negatively regulating the induction of autophagy, consistent with what has been shown during starvation-induced autophagy. Consistent with this, overexpression of Tor resulted in reduced autophagy with delayed midgut PCD (Supplementary Figure S4).

TOR can assemble into distinct complexes, TORC1 and TOCR2, with Raptor and Rictor, respectively. TORC1 is well established as a negative regulator of autophagy. ${ }^{42}$ Consistent with this role of TORC1, knockdown of raptor resulted in earlier autophagy induction and smaller midguts, whereas rictor knockdown had no effect (Figures 7a-f). The knockdown of Tor or raptor in the fat body was sufficient to induce autophagy under fed conditions that was not altered by starvation, whereas the knockdown of rictor showed robust induction of autophagy following starvation (Figures $7 \mathrm{~g}-\mathrm{j}$ ). These results suggest that the negative control of autophagy by TORC1 during midgut removal is similar to the role of TORC1 during starvation-induced autophagy in the fat body. ${ }^{41}$

To examine the requirement of Atg1 downstream of Tor during midgut removal, we examined the combined knockdown of Atg1 and Tor. Knockdown of Tor showed shorter gastric caeca at an earlier but not later time point (Figure 8b) and autophagy was induced at an earlier stage (Figures 7b and 8b). Knockdown of Atg1 showed larger gastric caeca at both early and late times, accompanied by reduced autophagy (Figures 8c, e and f). Simultaneous knockdown of both Atg1 

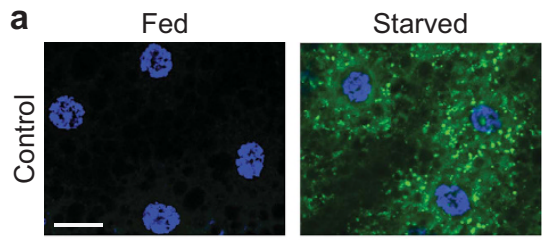

b

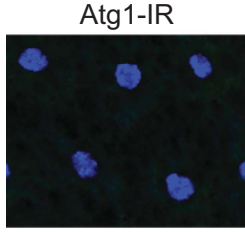

Atg13-IR
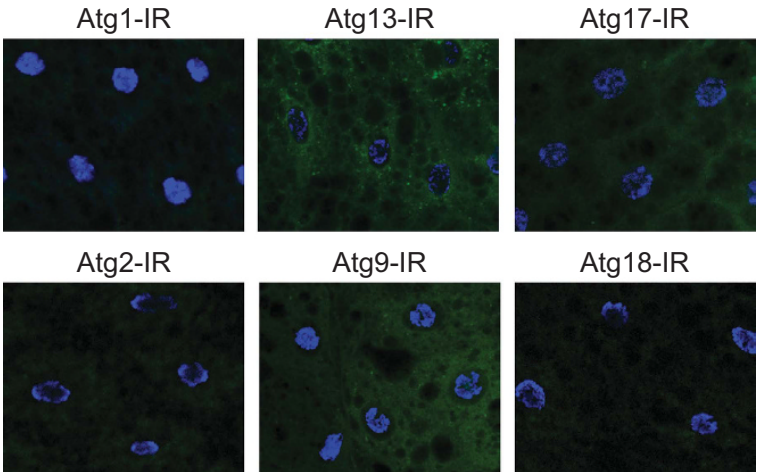

Atg3-IR

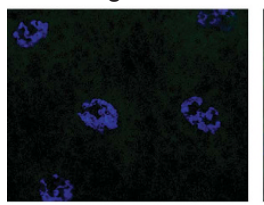

Atg5-IR

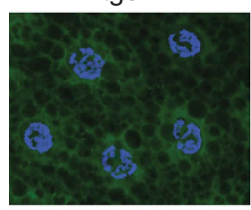

Atg6-IR

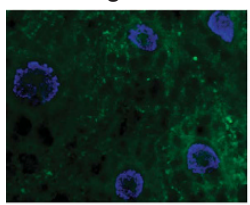

Atg14-IR
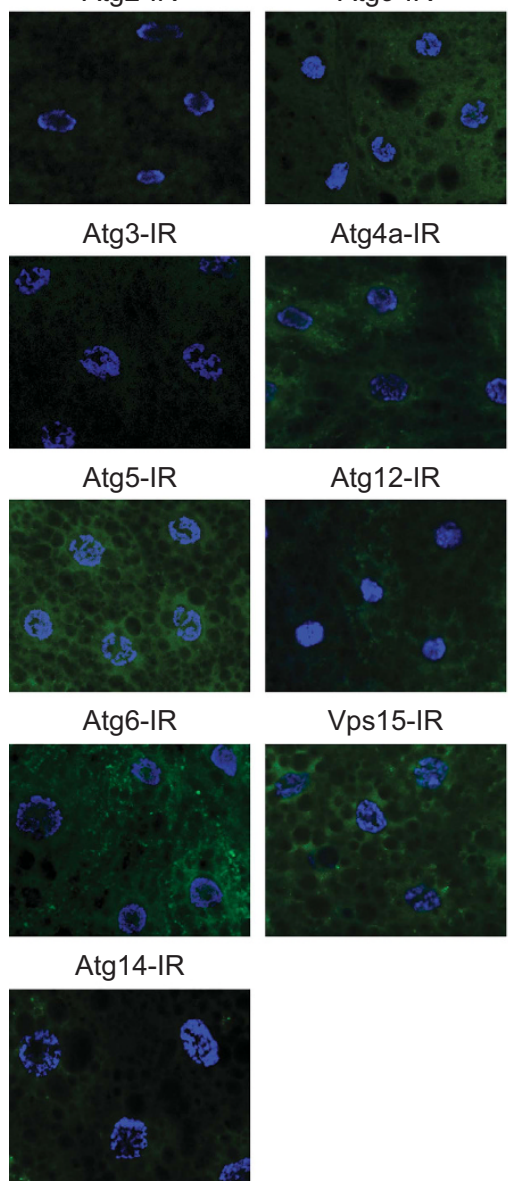

Atg4a-IR

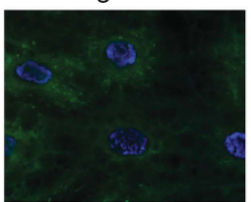

Atg12-IR

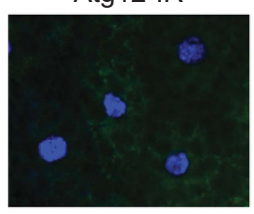

Vps15-IR
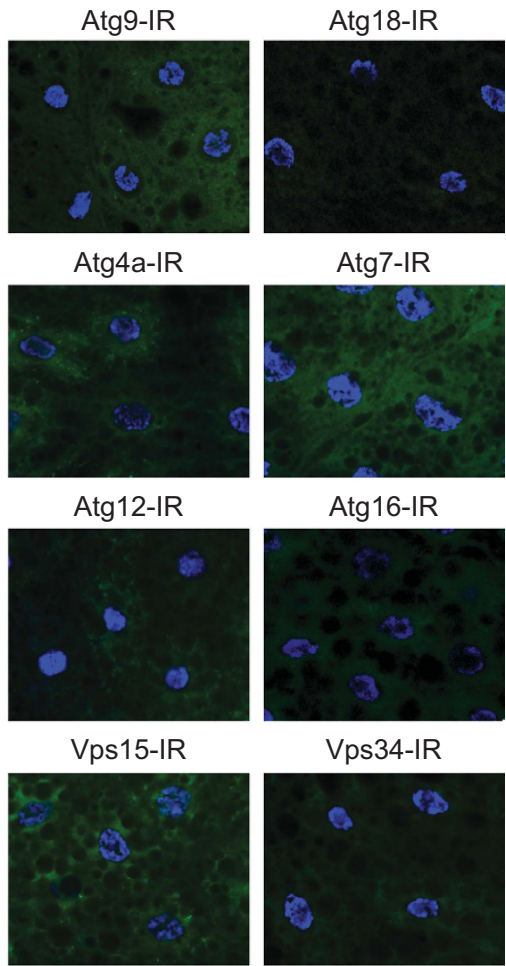

Atg7-IR

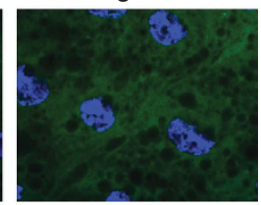

Atg16-IR

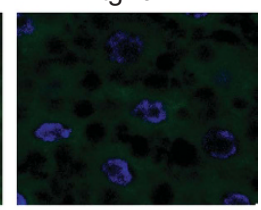

Vps34-IR

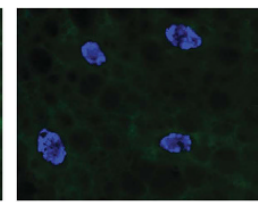

Atg8a-IR

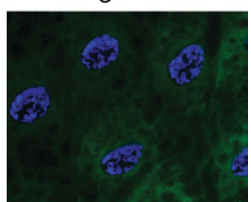

Figure 6 Atg gene knockdown prevents starvation-induced autophagy in the fat body. Lysotracker staining of fat body from larvae at $96 \mathrm{~h}$ after egg deposition (AED) starved for $2 \mathrm{~h}$. (a) Control (CG-GAL4/+) shows low levels of lysotracker puncta in fat body from fed larvae (a, left) that increases following starvation (a, right). (b) Following starvation, fat body from Atg gene knockdown all showed very low levels of lystoracker puncta compared with starved control (a, right). DNA is stained by Hoechst (blue). Scale bar represents $10 \mu \mathrm{m}$

and Tor restored autophagy and gastric caeca contraction to levels similar to those seen in control (Figures $8 d-f)$. Together this implies that in the absence of Tor, Atg1 is not essential or low levels are sufficient for induction of autophagy and suggests that Tor may act in multiple pathways to inhibit autophagy.

\section{Discussion}

Our work provides a detailed analysis of the requirements for canonical autophagy components in regulation of Drosophila larval midgut PCD. We show that there is a distinct requirement for specific components for midgut autophagy and PCD that differs from autophagy induced in the fat body in response to starvation. We also demonstrate that, despite requiring specific Atg genes, the upstream regulation of autophagy by TORC1 in the midgut appears to be similar to that regulating starvation-induced autophagy. Surprisingly though, our data suggest that, in the absence of Tor, Atg1 is not essential for autophagy induction. This implies that an alternative mechanism regulating autophagy induction may exist, and it will be important to dissect out this pathway in future studies.

We have shown that the components of the initiation complex are essential for induction of autophagy and midgut degradation. Depletion of Atg1, Atg13, Atg17 or Atg101 blocked autophagy induction and dramatically delayed midgut removal. These finding are consistent with the conserved role of this complex in autophagy induction in other systems.

The class III PI3K complex, consisting of Atg6, Vps15 and Vps34, contributes to autophagsome formation, by recruiting phosphatidylinositol 3-phosphate and other proteins (such as Atg18). ${ }^{35,46}$ Consistent with our result in the fat body with Atg6 knockdown, the loss-of-function Atg6 mutants shows disruption of autophagy in the fat body. ${ }^{33,41}$ Surprisingly, knockdown of Atg6 did not reduce autophagy in midgut yet delayed midgut histolysis. This could suggest that Atg6 has an alternative role in midgut degradation other than autophagy. Atg6 is required for starvation-induced autophagy in the fat body and also has roles in vesicle trafficking and haematopoiesis. ${ }^{33}$ This multifunction of the PI3K complex may explain the different requirements for Vps15 and Vps34 in midgut removal and autophagy. ${ }^{48}$ Other autophagy-independent processes of Vps15 and Vps34 include regulation of protein secretion and other vesicular trafficking processes, including protein sorting. ${ }^{49}$

Our data suggest that there are different requirements for the two conjugation pathways during midgut PCD. Although Atg8a has an essential role in midgut removal, the components required for lipidation are not essential. An alternative conjugation pathway has been proposed, and that the function of Atg8a is independent of its lipidation thus Atg 3 and Atg7 function is not required..$^{23}$ However, the molecular mechanism of this pathway remains to be established. The E3-like complex consisting of Atg5 and Atg12 promotes the transfer of Atg8 from Atg3 to PE and facilitates autophagosome expansion. The knockdown of Atg5, Atg12 or Atg16 resulted in a moderate delay in midgut degradation, with no significant effect on autophagy. This is in contrast to the essential role of these components in starvationinduced autophagy and suggests that an alternative conjugation pathway may compensate.

The molecular functions of Atg2 and Atg9 have not been characterised in Drosophila. The yeast orthologues function in lipid and protein recycling and assembly of autophagic membrane. ${ }^{43}$ Additionally, the initiation of autophagy requires direct phosphorylation of Atg9 by Atg1, which is required for recruitment of Atg8 and Atg18 to the autophagosome 

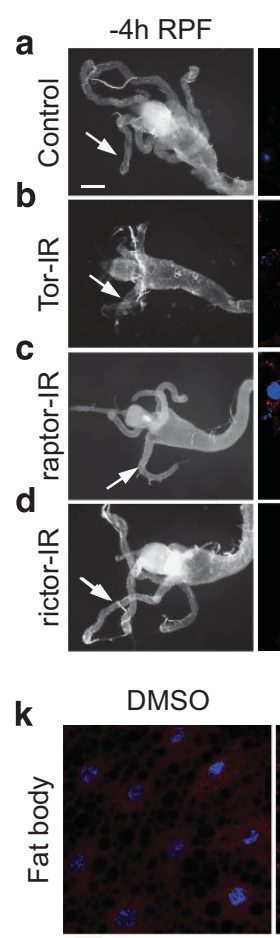

e
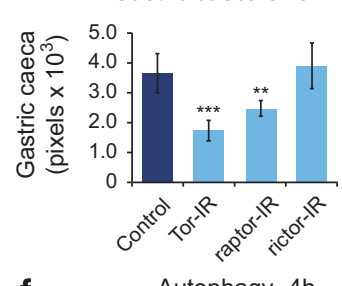

f

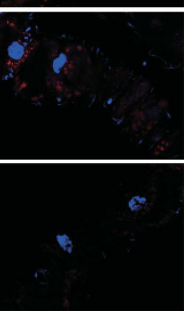

Rapamycin
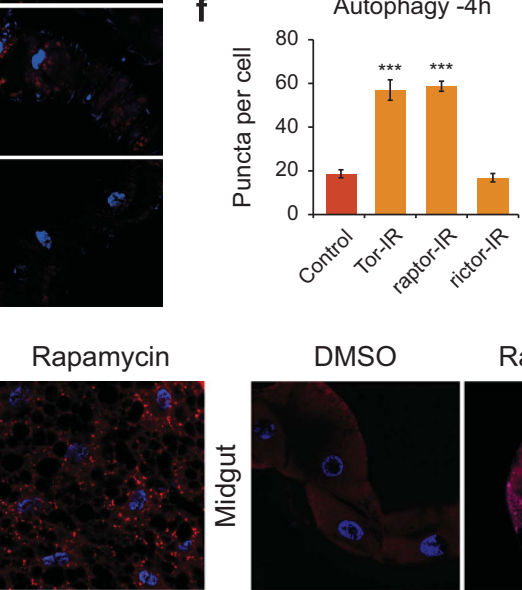

Gastric caeca size $-4 \mathrm{~h}$
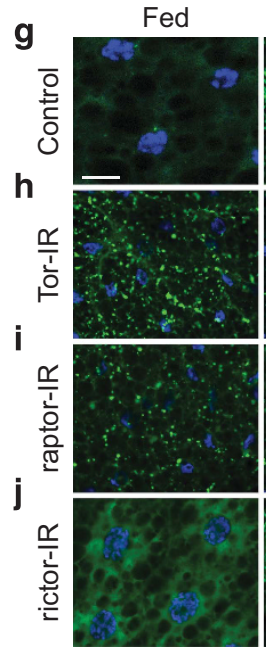

Starved
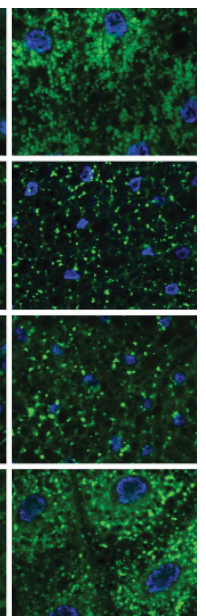

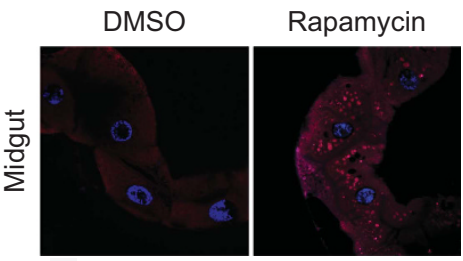

Figure 7 Knockdown of Tor and raptor, but not rictor, induces premature autophagy and degradation of midgut. Compared with (a) control (NP1-GAL4/+; pmCherry-Atg8a/+), morphological analysis shows that midguts of (b) Tor-IR (NP1-GAL4/+; UAS-Tor RNAi/pmCherry-Atg8a) and (c) raptor-IR (NP1-GAL4/+; UAS-raptor RNAi/pmCherry-Atg8a) have shortened gastric caeca at $-4 \mathrm{~h}$ RPF, whereas (d) rictor-IR (NP1-GAL4/+; UAS-raptor RNAi/pmCherry-Atg8a) was similar to the control (arrow). Scale bar represents $200 \mu \mathrm{m}$. (e) Quantitation of gastric caeca size (average pixels \pm S.D.) at $-4 \mathrm{~h} \mathrm{RPF}$ from (a-d) $\left(\mathrm{N} \geq 10,{ }^{* \star \star} P<0.001,{ }^{* \star} P<0.01\right)$. At an early time point $(-4 \mathrm{~h} \mathrm{RPF})$ autophagy levels are low in control (a, right), and are increased in Tor-IR and raptor-IR (b and c, right). (f) Quantitation of mCherry-Atg8a puncta at $-4 \mathrm{~h} \mathrm{RPF}$ (average puncta/cell \pm S.E.M. $\left.{ }^{* \star \star} P<0.001\right)$. Compared with (g) control (CG-GAL4/+), fat body of (h) Tor-IR (CG-GAL4/+;UAS-Tor RNAi/+) and (i) raptor-IR (CG-GAL4/+;UAS-raptor RNAi/++) showed higher levels of autophagy under feeding condition (left) but not enhanced when starved (right). In fat body of (j) rictor-IR (CG-GAL4/+,UAS-rictor RNAi/+), level of autophagy was similar to control fed (left) or starved (right). (k) High-dose of rapamycin $(400 \mu \mathrm{M})$ induces autophagy in fat body and midgut. Both fat body (left) and midgut (right) of larvae fed with $400 \mu \mathrm{M}$ rapamycin show robust lysotracker-positive puncta compared with the control fed with dimethyl sulphoxide (DMSO)

formation site and expansion of the isolation membrane. ${ }^{50}$ Our data show that knockdown of Atg2, Atg9 or Atg18 result in a similar delay in midgut degradation and disruption of autophagy. Combined with earlier observations, ${ }^{17,19}$ our data here suggest that the Atg2, Atg9 and Atg18 complex is essential for the initiation of autophagy in the midgut, rather than only working as a recycling mechanism.

Although the components of the autophagy pathway required for midgut degradation differ to that required for starvation-induced autophagy in the fat body, we demonstrate that in both cases TORC1 negatively regulates the initiation of autophagy. The level of autophagy and extent of midgut degradation were found to be directly correlated with TORC1 function. Consistent with this, raptor depletion resulted in premature autophagy similar to Tor ablation, suggesting that raptor and Tor function in TORC1 and negatively regulates autophagy in this cell death context.

Our study shows that distinct components of the core autophagy machinery are required during midgut $\mathrm{PCD}$ (Supplementary Figure S5). Furthermore, the findings here reveal that while some Atg proteins are not required for autophagosome formation they are required for the cell size reduction that occurs during midgut histolysis. This intriguing finding suggests that alternative pathways of autophagy function in specific tissues with the potential for autophagy- independent roles of Atg proteins. It is likely that the different forms of autophagy may require distinct regulatory components and this may be based on specific initiating signals. Autophagy can be regulated by numerous pathways, and while most centre around TOR activity, TOR-independent regulation can also occur. It will be important to determine the nature of the upstream signalling during autophagydependent midgut PCD as well as determining the nonautophagic function of Atg genes. It is possible that distinct forms of autophagy are required depending on the rate of autophagy required, or if it needs to be maintained for an extended time and the cellular outcome (whether the engulfed material is being recycled or degraded). Our data shows that the cargo receptor p62 is not essential for autophagy. This suggests that the nature of the cytoplasmic targets may have a role, whether non-selective bulk degradation of cytoplasmic targets is required or whether specific cargo are selected. All these factors may contribute to context-specific requirements for distinct autophagy machinery.

Overall, as summarised in Supplementary Figure S5, our study suggests that somewhat different mechanisms execute autophagy in dying midguts and fat body under starvation. Further studies will be required to fully delineate the regulation and mechanisms that mediate autophagydependent cell death in the midgut and to understand what 
a

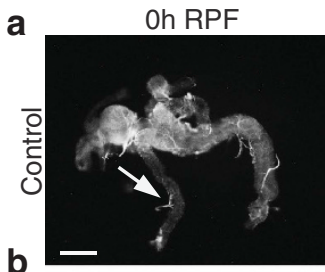

b

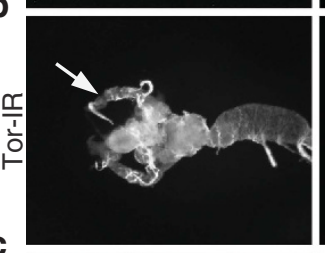

C

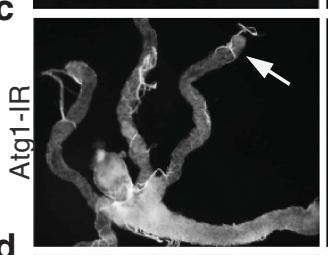

d

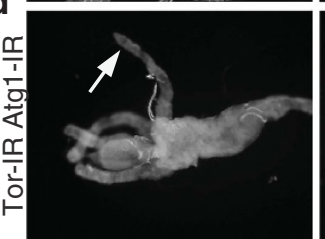

e

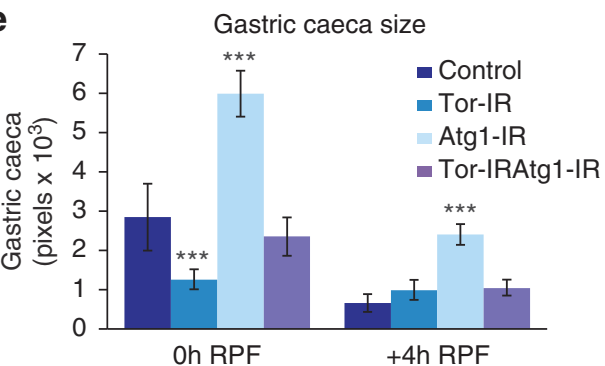

+4h RPF
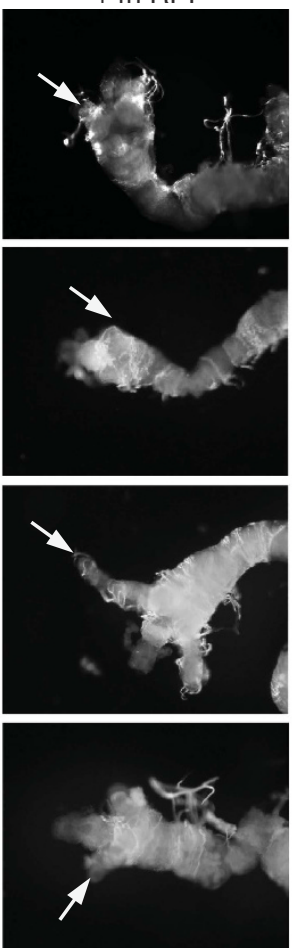

+4h RPF +2h RPF
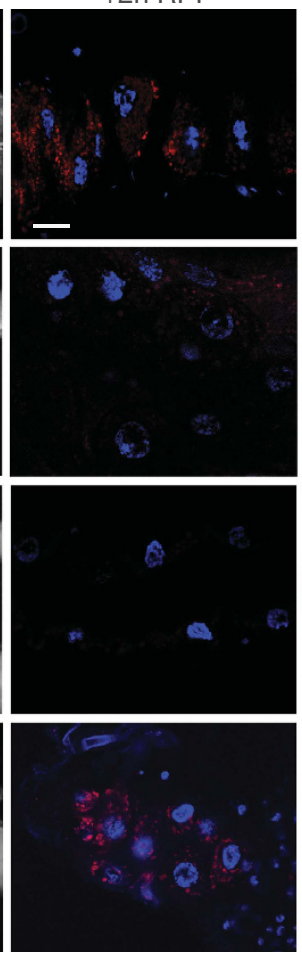

f

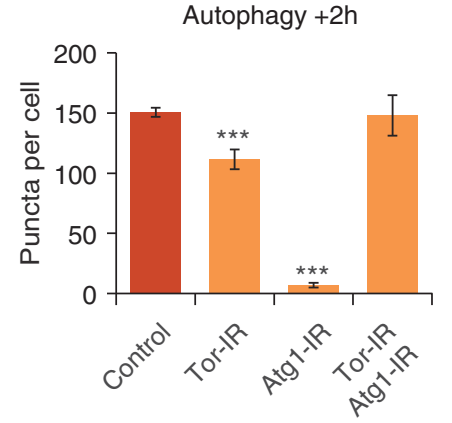

$+2 \mathrm{~h}$ RPF
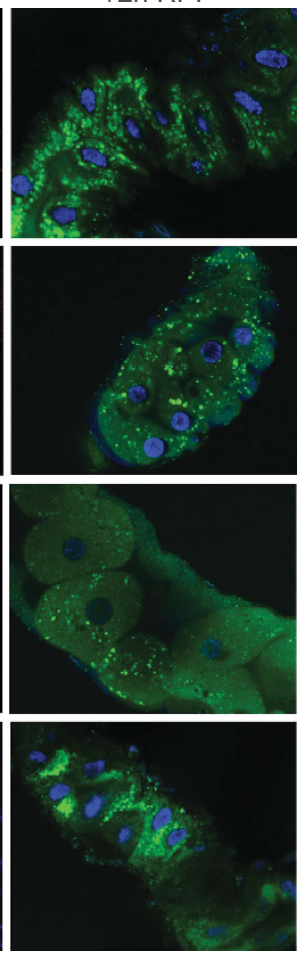

Autophagy $+2 h$

Figure 8 Tor and Atg1 double knockdown retains wild-type phenotype. (a-d, left side) Morphology of midguts at $0 \mathrm{~h}$ and $+4 \mathrm{~h}$ RPF. Scale bar represents $200 \mu \mathrm{m}$. (a-d, right side) Autophagy induction at $+2 \mathrm{~h}$ RPF monitored by pmCherry-Atg8a (red) and lystoracker (green) puncta. DNA is stained by Hoechst (blue). Scale bar represents $10 \mu \mathrm{m}$. Compared with (a) control (NP1-GAL4/+; pmCherry-Atg8a/+), the delayed midgut degradation and low autophagy in (c) Atg1-IR (NP1-GAL4/+; UAS-Atg1 RNAi/pmCherry-Atg8a) is rescued by co-knockdown of (d) Tor-IR (NP1-GAL4/pmCherry-Atg8a;UAS-Atg1 RNAi/UAS-Tor RNAi \#1). (b) Tor-IR (NP1-GAL4/+;UAS-Tor RNAi \#1/pmCherry-Atg8a) with smaller midguts. (e) Quantitation shows that gastric caeca (arrow) size of the double knockdown was not significantly different compared with control (average pixels \pm S.D., $N \geq 10,{ }^{* *} P<0.005$ ). (f) Quantitation shows that the combined knockdown of Atg1 and Tor restores autophagy to levels similar to control (average pixels S.E.M., $N \geq 10$, $\left.{ }^{\star \star \star} P<0.005\right)$

makes autophagy required for PCD distinct from starvationinduced autophagy.

\section{Materials and Methods}

Fly stocks. We used midgut driver NP1-GAL4, obtained from the Drosophila genetic resource center (Kyoto, Japan) crossed to UAS-LacZ RNAi (obtained from Kennerdell and Carthew ${ }^{51}$ ) or $w^{1118}$ (Bloomington Stock Center, Bloomington, IN, USA) as control. To drive expression in fat body, CG-GAL4 was used. To monitor autophagy induction, we used pmCherry-Atg8 $\mathrm{a}^{52}$ and UAS-eGFP-Atg5 (Bloomington Stock Center). The lines to knockdown Atg genes, UAS-RNAi, were obtained from the Bloomington Stock Center or Vienna Drosophila RNAi Center (Vienna, Austria) as listed in Table 1. All flies were maintained and crossedls performed at $25{ }^{\circ} \mathrm{C}$ on cornmeal, molasses and yeast medium. Larvae were grown on food containing $0.05 \%$ bromophenol blue and staged by collection of wandering clear gut larvae onto damp Whatmann paper in a petri dish as previously described. ${ }^{17,52}$
Detection and quantitation of autophagy in the midgut. Because of a lack of suitable reagents for monitoring autophagy flux, Atg8a puncta is commonly used for detecting autophagy in vivo in Drosophila. ${ }^{53}$ Autophagy was assessed by pmCherryAtg8a puncta formation. ${ }^{52}$ Additionally, eGFP-Atg5 puncta was also used to measure autophagy. ${ }^{17}$ In the case of Atg8a knockdown, eGFP-Atg5 was used as the autophagy level indicator to avoid a false result. Midguts from pupae staged +2 h RPF were dissected in PBS, stained with Hoechst 33342 (Sigma-Aldrich, St. Louis, MO, USA) and imaged immediately without fixation using Zeiss LSM-700 (Jena, Germany) confocal microscope. From at least 10 confocal images for each genotype, ImageJ (Bethesda, MD, USA) was used to count puncta with a size $>2$ pixels for all experiments unless otherwise stated in the selected channel and represented as the average puncta per cell. Images of $A \operatorname{tg} 8 a^{K G 07569}$ Figure $4 \mathrm{C}$ and the control group plotted in Figure $4 f$ with $A \operatorname{tg} 8 a^{K G 07569}$ were taken using a Bio-Rad confocal microscope (Hercules, CA, USA) as previously described. ${ }^{17}$ Due to the background on Bio-Rad confocal images, particle $>4$ pixels were counted in ImageJ. 
Table 1 Genotypes and stock number

\begin{tabular}{|c|c|c|}
\hline Gene & Line & Genotype \\
\hline Atg1 & BL26731 & $y^{1} v^{1} ; P\{T R i P . J F 02273\} a t t P 2$ \\
\hline Atg2 & BL27706 & $y^{1} v^{1}: P\{$ TRIP.JF02786\}attP2 \\
\hline $\operatorname{Atg} 3$ & V22455 & $w^{1118} ; P\{G D 12064\} V 22455$ \\
\hline Atg $4 a$ & $\begin{array}{l}\text { BL34359 } \\
\text { V34843 }\end{array}$ & $\begin{array}{l}y^{1} s C^{*} v^{1} ; P\{\text { TriP.HMS01348\}attP2 } \\
w^{1118} P\{G D 11315\} 34843\end{array}$ \\
\hline & BL28367 & $y^{1} v^{1} ; P\{$ TriP.JF03003\}attP2 \\
\hline & BL35740 & $y^{1} s c^{*} v^{1} ; P\{$ TriP.HMS01482\}attP2 \\
\hline Atg5 & $\begin{array}{l}\text { BL27551 } \\
\text { BL34899 }\end{array}$ & $\begin{array}{l}y^{1} v^{1} ; P\{\text { TriP.JF02703\}attP2 } \\
y^{1} s c^{*} v^{1}: P\{\text { TRIP.HMS01244\}attP2 }\end{array}$ \\
\hline Atg6 & $\begin{array}{l}\text { BL28060 } \\
\text { BL35741 }\end{array}$ & $y^{1} v^{1} ;$ P TriP.JFO2897\}attP2 \\
\hline Atg7 & $\begin{array}{l}\text { BL34369 } \\
\text { BL27707 } \\
\text { V45558 }\end{array}$ & $\begin{array}{l}y^{1} s c^{*} v^{1} ; P\left\{\text { TriP.HMS01358\}attP2/TM3, } S b^{1}\right. \\
y^{1} v^{1} ; P\{\text { TriP.JF02787\}attP2 } \\
w^{1118} ; P\{G D 11671\} \text { v45558 }\end{array}$ \\
\hline Atg8a & $\begin{array}{l}\text { v43097 } \\
\text { BL34340 } \\
\text { BL14639 }\end{array}$ & $\begin{array}{l}w^{1118} ; P\{G D 4654\} \text { V43097 } \\
y^{1} s c^{*} v^{1} ; P\{\text { TriP.HMS01328\}attP2 } \\
y^{1} \text { Atg8a } a^{\text {KGO7569/FM7c }}\end{array}$ \\
\hline Atg $8 b$ & $\begin{array}{l}\text { BL27554 } \\
\text { BL34900 }\end{array}$ & $\begin{array}{l}y^{1} v^{1} ; P\{\text { TriP.JF02706\}attP2 } \\
y^{1} s c^{*} v^{1} ; P\{\text { TriP.HMS01245\}attP2 }\end{array}$ \\
\hline $\operatorname{Atg} 9$ & $\begin{array}{l}\text { BL28055 } \\
\text { BL34901 }\end{array}$ & $\begin{array}{l}y^{1} v^{1} ; P\{\text { TriP.JFO2891\}attP2 } \\
y^{1} s c^{*} v^{1} ; P\{\text { TriP.HMS01246\}attP2 }\end{array}$ \\
\hline Atg12 & $\begin{array}{l}\text { v29790 } \\
\text { BL27552 } \\
\text { BL34675 }\end{array}$ & $\begin{array}{l}w^{1118} ; P\{G D 15230\} v 29790 / C y O \\
y^{1} v^{1} ; P\{\text { TRiP.JF02704\}attP2 } \\
y^{1} s c^{*} v^{4} ; P\{\text { TRiP.HMS01153\}attP2 }\end{array}$ \\
\hline $\operatorname{Atg} 13$ & BL40861 & $y^{1} v^{1} ; P\{$ TRIP.HMS02028\}attP40 \\
\hline Atg14 & BL55398 & $y^{1} v^{1} ; P\{$ TRiP.HMC04086\}attP2 \\
\hline Atg16 & BL34358 & $y^{1} s c^{*} v^{1} ; P\{$ TRiP.HMS01347\}attP2 \\
\hline Atg17 & BL36918 & $y^{1} s c^{*} v^{1} ; P\left\{\right.$ TriP.HMS01611\}attP2/TM3, Sb ${ }^{1}$ \\
\hline Atg18 & $\begin{array}{l}\text { v22646 } \\
\text { BL28061 }\end{array}$ & $\begin{array}{l}\text { w1118; P\{GD12342\}v22646/TM3 } \\
y^{1} v^{1} ; P\{T R i P . J F 02898\} a t t P 2\end{array}$ \\
\hline Atg101 & BL34360 & $y^{1} s c^{*} v^{4} ; P\{T R i P . H M S 01349\} a t t P 2$ \\
\hline Vps 15 & $\begin{array}{l}\text { BL34092 } \\
\text { BL35209 }\end{array}$ & $\begin{array}{l}y^{1} s c^{*} v^{1} ; \text { P }\{\text { TRIP.HMS00908\}attP2 } \\
y^{1} s c^{*} v^{1} ; \text { P }\{\text { TRIP.GL00085\}attP2 }\end{array}$ \\
\hline Vps34 & $\begin{array}{l}\text { BL33384 } \\
\text { BL36056 }\end{array}$ & $\begin{array}{l}y^{1} s c^{*} v^{1} ; P\left\{\text { TRiP.HMS00261\}attP2/TM3, } S b^{1}\right. \\
y^{1} s c^{*} v^{1} ; \text { P }\left\{\text { TRiP.GL00175\}attP2/TM3, } S b^{1}\right.\end{array}$ \\
\hline $\operatorname{ref}(2) P$ & $\begin{array}{l}\text { BL33978 } \\
\text { BL36111 }\end{array}$ & $\begin{array}{l}y^{1} s c^{*} v^{1} ; P\{\text { TRIP.HMS00938\}attP2 } \\
y^{1} s c^{*} v^{1} ; P\{\text { TRIP.HMS00551\}attP2 }\end{array}$ \\
\hline Tor & $\begin{array}{l}\text { BL33951 } \\
\text { BL35578 } \\
\text { BL7012 }\end{array}$ & $\begin{array}{l}y^{1} s c^{*} v^{1} ; P\{\text { TRiP.HMS00904\}attP2 } \\
y^{1} s c^{*} v^{1} ; P\{\text { TRiP.GLO0156\}attP2 } \\
y^{1} w^{*} P\{r y[+t 7.2]=h s F L P\} 12 ; P\{w[+m C]=U A S-T o r . W T\}\end{array}$ \\
\hline & $\begin{array}{l}\text { BL31529 } \\
\text { BL34814 }\end{array}$ & $\begin{array}{l}y^{1} v^{1} ; P\{\text { TRiP.JF01088\}attP2 } \\
y^{1} s c^{*} v^{1} ; P\{\text { TRiP.HMS00124\}attP2 }\end{array}$ \\
\hline rictor & $\begin{array}{l}\text { BL36584 } \\
\text { BL36699 }\end{array}$ & $\begin{array}{l}y^{1} S c^{*} v^{1} ; P\{\text { TRIP.GLO0544\}attP40 } \\
y^{1} S c^{*} v^{1} ; \text { P }\{\text { TRIP.HMS01588\}attP2 }\end{array}$ \\
\hline
\end{tabular}

Midgut morphology and quantitation. Animals were staged $-4 \mathrm{~h}, \mathrm{Oh}$ or $+4 \mathrm{~h} \mathrm{RPF}$, and midguts were dissected in PBS, fixed in $4 \%$ formaldehyde/PBS and examined using a stereozoom microscope (Olympus, Tokyo, Japan). Measurements of gastric caeca size were done using Photoshop (Adobe, San Jose, CA, USA) magnetic lasso tool. The histogram function was used to determine pixels included in the area as previously described, including at least 10 midguts in each sample. ${ }^{52}$

Histology. Whole pupae staged to $+12 \mathrm{~h}$ RPF were fixed in FAAG ( $85 \%$ ethanol, $4 \%$ formaldehyde, $5 \%$ acetic acid and $1 \%$ glutaraldehyde), paraffin embedded before sectioning and then haematoxylin and eosin stained as previously described. ${ }^{17}$ For each genotype, a minimum of 10 pupae were examined using a stereozoom microscope.

Autophagy in fat body. Larvae were aged in normal food $96 \mathrm{~h}$ after egg deposition (AED) and transferred to petri-dish for $2 \mathrm{~h}$ starvation. The fat body was dissected in PBS with $1 \mu \mathrm{M}$ LysoTracker Green DND 26 (Invitrogen, Carlsbad, CA, USA) and Hoechst 33342.

Imaging. Confocal images were obtained using a confocal microscope with Argon ion $488 \mathrm{~nm}(14 \mathrm{mw})$ and Green HeNe $543 \mathrm{~nm}(1.5 \mathrm{mw})$ lasers and a Zeiss inverted microscope with a $40 \times$ UPLAPO $(N A=1.2$ water) objective. The duallabelled samples were imaged with two separate channels (PMT tubes) in a sequential setting. Green fluorescence was excited with an Ar $488 \mathrm{~nm}$ laser line, and the emission was viewed through a HQ515/30 nm narrow band barrier filter in PMT1. Red fluorescence was excited with a HeNe $543 \mathrm{~nm}$ laser line, and the emission was viewed through a long pass barrier filter (E570LP) in PMT2. Automatically, all signals from PMTs 1 and 2 were merged. Images were captured using the Zen (Jena, Germany) software and compiled using Photoshop CS5 (Adobe). In all experiments, samples were immediately imaged with a laser-scanning confocal microscope (Zeiss LSM-700) unless otherwise stated.

Rapamycin feeding. Larvae were aged in normal food $96 \mathrm{~h}$ after egg deposition (AED) and transferred to food containing the indicated concentration of rapamycin for $2 \mathrm{~h}$ (original stock $1 \mathrm{mM}$ dissolved in DMSO). 0.05\% Bromophenol blue was also added to the food to ensure uptake of treated food that would appear blue in the gut. Food containing same volume of DMSO was used as control treatment. Lysotracker is also widely used as an autophagy-level indicator especially in Drosophila fat body. ${ }^{41}$ We have also shown that lysotracker and pmCherryAtg8a co-localize in dying midgut (Supplementary Figure S3). Thus in the rapamycin experiments lysotracker was used as an indicator. The fat body and midguts were dissected in PBS with $1 \mu \mathrm{M}$ LysoTracker Red DND 99 and Hoechst 33342.

Quantitative real-time PCR. Quantitation of the level of RNAi knockdown of various lines was done by RT-qPCR. Total RNA was isolated from Drosophila midguts using TRlzol reagent (Invitrogen) and CDNA was synthesized from $1 \mu \mathrm{g}$ of total RNA using High Capacity cDNA Reverse Transcription Kit (Applied Biosciences, Foster City, CA, USA) and oligo dT primer. Real-Time qPCR was performed on a Rotor-Gene 6000 (Corbett Research, Mortlake, NSW, Australia) using $\mathrm{RT}^{2}$ Real-Time SYBR Green/ROX PCR MasterMix (Qiagen, Valencia, CA, USA) as per the manufacturer's instructions. Reactions were performed in triplicate, and the mRNA expression levels were normalized against the internal control gene rp49 using the $\triangle \triangle C T$ method. Primer sets used were:

rp49 F CCAGTCGGATCGATATGCTAA; R ACGTTGTGCACCAGGAACTT

Atg1 F ACGGCGGACAAGATTCTCTA; R GCTGCTGCAATATGCTCAAA

Atg2 $\mathrm{F}$ AAGTGGAAGCAGGAGATCCA; R AATCACACCCATGTCGGAAT

Atg3 F GCAATGTTCCCTGCTATCGT; R TGTCGCTATCTGGAGTGTGC

Atg 4 F CTTCTCCCTGCACTCGTTTC; R CTCGGTTTGATGGGATGACT

Atg6 $\mathrm{F}$ TTCCAGAAGGAGGTCGAGAA; R TCCCCATTTCAGGTTGGTTA

Atg7 F GGAATGCTGTGCAACTACGA; R GGAGATTCCCGTCAAATCCT

Atg8a F CATCGGTGATTTGGACAAGA; R TGCCGTAAACATTCTCATCG

Atg8b F TCAGTACCAGCAGAAGCACG; R AAGGCAAGTAGCCAGACGAC

Atg9 $\mathrm{F}$ CGGAGCTGCACAAGAAGAAG; R CTTGTCGAAGTGCTCCTGGT

Atg12 F CAATGTGCCCATCATCAAAA; R CACGCCTGATTCTTGCAGTA

Atg13 F ACAGCCACATATCGTCACCA; R GGTCGAGGAAATCGATGAGA

Atg14 F GTCAGAGGACGAAAACTCTC; R ATGGTAGACTGCTGGTTGG Atg16 F ATGCATCGATGTGCCGTGA; R CTTGCTTGCAGGATTCGTGG

Atg17 F CGTCTCCCTTCTTTGAGTCG; R CCAAACGTGGAGCCATTAGT Atg18 F AGGTGACCGACGTGTTTAGC; R ACGGTGGGAATGGAATACAC Atg101 F CGAGAACGAGGATGAGAGGC; R ACGTCCGGAAAGGATGTGTC Vsp15/ird1 F GAGCCGTAGTCCTTGGAGTG; R TGTCACCAGCTTGTCGTAGC Vsp34/PI3K59F F AGCCAATCTCTGCACATTCC; R GCCTCTCAGGTTGAAGTTGC ref(2)P F AAACCACCACCGAAACAGAG; R CCTCCTCATTCATGTGCTGA rictor $\mathrm{F}$ GCAGCCAATGAGTTACAGCA; R GAGCGTAGCTGGACATAGCC raptor F TCGGTTCTTTGAGGGAGCTA; R CGGCTGTCTCTCGTACATCA and Tor F CGGTTATCCCGCTCAGTACC; R GGTGATCATAGTCTGGCGCA

Statistical analysis of data. Student's t-test was used for all statistical analysis unless otherwise stated. Data are expressed as mean \pm S.D. or \pm S.E.M., as appropriate. $P<0.05$ was considered significant.

\section{Conflict of Interest}

The authors declare no conflict of interest.

Acknowledgements. This work was supported by the National Health and Medical Research Council of Australia Project Grant (1041807) and a Senior Principal Research Fellowship (1002863) to SK. TX was supported by a University of South Australia President's Scholarship and SN by an Australian Postgraduate Award. We thank Sonia Dayan, May Aung-Htut and other members of our laboratory for help and advice, the Australian Drosophila Research Support Facility, Vienna Drosophila RNAi Centre and Bloomington Drosophila Stock Center for Drosophila stocks and Amanda Rogers for tissue processing. 
1. Baehrecke EH. How death shapes life during development. Nat Rev Mol Cell Biol 2002; 3: 779-787.

2. Fuchs $Y$, Steller H. Programmed cell death in animal development and disease. Cell 2011; 147: $742-758$.

3. Galluzzi L, Vitale I, Abrams JM, Alnemri ES, Baehrecke EH, Blagosklonny MV et al. Molecular definitions of cell death subroutines: recommendations of the Nomenclature Committee on Cell Death 2012. Cell Death Differ 2012; 19: 107-120.

4. Denton D, Nicolson S, Kumar S. Cell death by autophagy: facts and apparent artefacts. Cell Death Differ 2012; 19: 87-95.

5. Denton D, Xu T, Kumar S. Autophagy as a pro-death pathway. Immunol Cell Biol 2015; 93: 35-42.

6. Melendez A, Neufeld TP. The cell biology of autophagy in metazoans: a developing story. Development 2008; 135: 2347-2360.

7. Yang Z, Klionsky DJ. Mammalian autophagy: core molecular machinery and signaling regulation. Curr Opin Cell Biol 2010; 22: 124-131.

8. Scott RC, Juhasz G, Neufeld TP. Direct induction of autophagy by Atg1 inhibits cell growth and induces apoptotic cell death. Curr Biol 2007; 17: 1-11.

9. Cecconi F, Levine B. The role of autophagy in mammalian development: cell makeover rather than cell death. Dev Cell 2008; 15: 344-357.

10. Mizushima N, Levine B, Cuervo AM, Klionsky DJ. Autophagy fights disease through cellular self-digestion. Nature 2008; 451: 1069-1075.

11. Kim DH, Sarbassov DD, Ali SM, King JE, Latek RR, Erdjument-Bromage $\mathrm{H}$ et al. mTOR interacts with raptor to form a nutrient-sensitive complex that signals to the cell growth machinery. Cell 2002; 110: 163-175.

12. Fang $Y$, Vilella-Bach $M$, Bachmann R, Flanigan A, Chen J. Phosphatidic acid-mediated mitogenic activation of mTOR signaling. Science 2001; 294: 1942-1945.

13. Frias MA, Thoreen CC, Jaffe JD, Schroder W, Sculley T, Carr SA et al. mSin1 is necessary for Akt/PKB phosphorylation, and its isoforms define three distinct mTORC2s. Curr Biol 2006; 16: 1865-1870.

14. Kim SG, Hoffman GR, Poulogiannis G, Buel GR, Jang YJ, Lee KW et al. Metabolic stress controls mTORC1 lysosomal localization and dimerization by regulating the TTT-RUVBL1/2 complex. Mol Cell 2013; 49: 172-185.

15. Peterson TR, Sengupta SS, Harris TE, Carmack AE, Kang SA, Balderas E et al. mTOR complex 1 regulates lipin 1 localization to control the SREBP pathway. Cell 2011; 146: 408-420.

16. Mammucari C, Milan G, Romanello V, Masiero E, Rudolf R, Del Piccolo $P$ et al. FoxO3 controls autophagy in skeletal muscle in vivo. Cell Metab 2007; 6: 458-471.

17. Denton D, Shravage B, Simin R, Mills K, Berry DL, Baehrecke EH et al. Autophagy, not apoptosis, is essential for midgut cell death in Drosophila. Curr Biol 2009; 19: $1741-1746$.

18. Denton D, Shravage B, Simin R, Baehrecke EH, Kumar S. Larval midgut destruction in Drosophila: not dependent on caspases but suppressed by the loss of autophagy. Autophagy 2010; 6: 163-165.

19. Berry DL, Baehrecke EH. Growth arrest and autophagy are required for salivary gland cell degradation in Drosophila. Cell 2007; 131: 1137-1148.

20. Denton D, Aung-Htut MT, Kumar S. Developmentally programmed cell death in Drosophila. Biochim Biophys Acta 2013; 1833: 3499-3506.

21. Denton D, Aung-Htut M, Lorensuhewa N, Nicolson S, Zhu W, Mills K et al. UTX coordinates steroid hormone mediated autophagy and cell death. Nat Commun 2013; 4: 2916.

22. Juhasz G, Erdi B, Sass M, Neufeld TP. Atg7-dependent autophagy promotes neuronal health, stress tolerance, and longevity but is dispensable for metamorphosis in Drosophila. Genes Dev 2007; 21: 3061-3066.

23. Chang TK, Shravage BV, Hayes SD, Powers CM, Simin RT, Wade Harper J et al. Uba1 functions in Atg7- and Atg3-independent autophagy. Nat Cell Biol 2013; 15: 1067-1078.

24. Mercer CA, Kaliappan A, Dennis PB. A novel, human Atg13 binding protein, Atg101, interacts with ULK1 and is essential for macroautophagy. Autophagy 2009; 5: 649-662.

25. Kabeya Y, Kamada Y, Baba M, Takikawa H, Sasaki M, Ohsumi Y. Atg17 functions in cooperation with Atg1 and Atg13 in yeast autophagy. Mol Biol Cell 2005; 16: 2544-2553.

26. Hara T, Takamura A, Kishi C, lemura S, Natsume T, Guan JL et al. FIP200, a ULK-interacting protein, is required for autophagosome formation in mammalian cells. J Cell Biol 2008; 181 : 497-510.

27. Hegedus K, Nagy P, Gaspari Z, Juhasz G. The putative HORMA domain protein Atg101 dimerizes and is required for starvation-induced and selective autophagy in Drosophila. Biomed Res Int 2014; 2014: 470482.
28. Nagy P, Karpati M, Varga A, Pircs K, Venkei Z, Takats S et al. Atg17/FIP200 localizes to perilysosomal Ref(2)P aggregates and promotes autophagy by activation of Atg1 in Drosophila. Autophagy 2014; 10: 453-467.

29. Chang YY, Neufeld TP. Autophagy takes flight in Drosophila. FEBS Lett 2010; 584: 1342-1349.

30. Kihara A, Noda T, Ishihara N, Ohsumi Y. Two distinct Vps34 phosphatidylinositol 3-kinase complexes function in autophagy and carboxypeptidase $Y$ sorting in Saccharomyces cerevisiae. J Cell Biol 2001; 152: 519-530.

31. Fogel Al, Dlouhy BJ, Wang C, Ryu SW, Neutzner A, Hasson SA et al. Role of membrane association and Atg14-dependent phosphorylation in beclin-1-mediated autophagy. Mol Cell Biol 2013; 33: 3675-3688.

32. Juhasz G, Hill JH, Yan Y, Sass M, Baehrecke EH, Backer JM et al. The class III PI(3)K Vps34 promotes autophagy and endocytosis but not TOR signaling in Drosophila. J Cell Biol 2008; 181: $655-666$

33. Shravage BV, Hill JH, Powers CM, Wu L, Baehrecke EH. Atg6 is required for multiple vesicle trafficking pathways and hematopoiesis in Drosophila. Development 2013; 140: 1321-1329.

34. Xie Z, Klionsky DJ. Autophagosome formation: core machinery and adaptations. Nat Cell Biol 2007; 9: 1102-1109.

35. Nakatogawa H, Suzuki K, Kamada Y, Ohsumi Y. Dynamics and diversity in autophagy mechanisms: lessons from yeast. Nat Rev Mol Cell Biol 2009; 10: 458-467.

36. Yang Z, Klionsky DJ. Eaten alive: a history of macroautophagy. Nat Cell Biol 2010; 12: 814-822.

37. Banreti A, Hudry B, Sass M, Saurin AJ, Graba Y. Hox proteins mediate developmental and environmental control of autophagy. Dev Cell 2014; 28: 56-69.

38. Erdi B, Nagy P, Zvara A, Varga A, Pircs K, Menesi D et al. Loss of the starvation-induced gene Rack1 leads to glycogen deficiency and impaired autophagic responses in Drosophila. Autophagy 2012; 8: 1124-1135.

39. Zheng YT, Shahnazari S, Brech A, Lamark T, Johansen T, Brumell JH. The adaptor protein p62 SQSTM1 targets invading bacteria to the autophagy pathway. J Immunol 2009; 183: 5909-5916.

40. Nezis IP, Simonsen A, Sagona AP, Finley K, Gaumer S, Contamine D et al. Ref(2)P, the Drosophila melanogaster homologue of mammalian p62, is required for the formation of protein aggregates in adult brain. J Cell Biol 2008; 180: 1065-1071.

41. Scott RC, Schuldiner O, Neufeld TP. Role and regulation of starvation-induced autophagy in the Drosophila fat body. Dev Cell 2004; 7: 167-178.

42. Webber JL, Tooze SA. Coordinated regulation of autophagy by p38alpha MAPK through mAtg9 and p38IP. EMBO J 2010; 29: 27-40.

43. He C, Baba M, Klionsky DJ. Double duty of Atg9 self-association in autophagosome biogenesis. Autophagy 2009; 5: 385-387.

44. Obara K, Sekito T, Ohsumi Y. Assortment of phosphatidylinositol 3-kinase complexesAtg14p directs association of complex I to the pre-autophagosomal structure in Saccharomyces cerevisiae. Mol Biol Cell 2006; 17: 1527-1539.

45. Obara K, Sekito T, Niimi K, Ohsumi Y. The Atg18-Atg2 complex is recruited to autophagic membranes via phosphatidylinositol 3-phosphate and exerts an essential function. J Biol Chem 2008; 283: 23972-23980.

46. Reggiori F, Klionsky DJ. Autophagosomes: biogenesis from scratch? Curr Opin Cell Biol 2005; 17: 415-422.

47. Diaz-Troya S, Perez-Perez ME, Florencio FJ, Crespo JL. The role of TOR in autophagy regulation from yeast to plants and mammals. Autophagy 2008; 4: 851-865.

48. Anding AL, Baehrecke EH. Vps15 is required for stress induced and developmentally triggered autophagy and salivary gland protein secretion in Drosophila. Cell Death Differ 2014; 22: 457-464.

49. Lindmo K, Stenmark H. Regulation of membrane traffic by phosphoinositide 3-kinases. J Cell Sci 2006; 119: 605-614.

50. Papinski D, Schuschnig M, Reiter W, Wilhelm L, Barnes CA, Maiolica A et al. Early steps in autophagy depend on direct phosphorylation of Atg9 by the Atg1 kinase. Mol Cell 2014; 53 : 471-483.

51. Kennerdell JR, Carthew RW. Heritable gene silencing in Drosophila using doublestranded RNA. Nat Biotechnol 2000; 18: 896-898.

52. Denton D, Chang TK, Nicolson S, Shravage B, Simin R, Baehrecke EH et al. Relationship between growth arrest and autophagy in midgut programmed cell death in Drosophila. Cell Death Differ 2012; 19: 1299-1307.

53. Nezis IP, Lamark T, Velentzas AD, Rusten TE, Bjorkoy G, Johansen T et al. Cell death during Drosophila melanogaster early oogenesis is mediated through autophagy. Autophagy 2009; 5: 298-302. 